Ian D. Watson*, Wytze P. Oosterhuis, Per E. Jorgensen, Z. Gunnur Dikmen, Joanna Siodmiak, Snezana Jovicic, Kristin M. Aakre, Vladimir Palicka and Marge Kutt, European Federation of Clinical Chemistry and Laboratory Medicine Working Group on Patient Focused Laboratory Medicine

\title{
A survey of patients' views from eight European countries of interpretive support from Specialists in Laboratory Medicine
}

DOI 10.1515/cclm-2017-0080

Received January 27, 2017; accepted April 2, 2017; previously published online May 5, 2017

\section{Abstract}

Background: There is increasing interest in direct patient engagement including receiving their laboratory medicine results. We previously established an appetite for Specialists in Laboratory Medicine to support patients in understanding results. The aim of this study was to establish whether patients agreed with such an approach, determined through surveying views in eight European countries.

Methods: A standardized five-question survey was administered across eight European countries to a total of 1084 individuals attending medical outpatient clinics, with 100 patients each in Poland, Serbia, Netherlands, Turkey and Czech Republic, 101 in Estonia, 116 in Denmark and 367 in Norway. The responses across countries were compared using the chi-square test $(\mathrm{p}<0.05)$.

*Corresponding author: Ian D. Watson, c/o EFLM Office, Via Carlo Farini 81, 20159 Milan, Italy, E-mail: iandwat@me.com

Wytze P. Oosterhuis: Department Clinical Chemistry and Haematology, Zuyderland Medical Center, Heerlen, The Netherlands Per E. Jorgensen: Hospital Management, Rigshospitalet, Copenhagen, Denmark

Z. Gunnur Dikmen: Department Biochemistry, Faculty of Medicine, Hacettepe University, Ankara, Turkey

Joanna Siodmiak: Department Laboratory Medicine, Faculty of Pharmacy, Collegium Medicum in Bydgoszcz, Nicolaus Copernicus University in Torun, Bydgoszcz, Poland

Snezana Jovicic: Department for Medical Biochemistry, Faculty of Pharmacy, Center for Medical Biochemistry, Clinical Center of Serbia, University of Belgrade, Belgrade, Serbia

Kristin M. Aakre: Laboratory of Clinical Biochemistry, Haukeland University Hospital, Bergen, Norway

Vladimir Palicka: Institute for Clinical Biochemistry and Diagnostics, Charles University and University Hospital Hradec, Kralove, Czech Republic

Marge Kutt: Diagnostic Clinic, Laboratory, North Estonian Medical Centre, Tallin, Estonia
Results: Patients wanting their results ranged from 50\% to $94 \%$ (mean $65 \%$ ) of those responding positively, a mean of $72 \%$ wanted additional information with their results; direct receipt was preferred over referral to a website. Specialists in Laboratory Medicine providing such information were acceptable to a mean of $62 \%$ of those respondents wishing their results; in countries where payment was possible, there was little interest in making additional payment for such a service.

Conclusions: A clear proportion of patients are interested in receiving their laboratory medicine results, the majority with explanatory notes; a role for Specialists in Laboratory Medicine is acceptable and raises the potential for direct engagement by such specialists with patients offering a new paradigm for the provision of laboratory medicine activities.

Keywords: patient engagement; patient support; patient understanding results; Specialist in Laboratory Medicine.

\section{Introduction}

Across Europe, there is an increasing trend for patients to have direct access to their medical record, including their laboratory test results, and although a systematic review of patient access to electronic records was unable to demonstrate a significant impact [1], this is the direction of travel. Although not yet as prevalent in Europe as in the USA, there are concerns over those for whom such an approach is a barrier. We have previously established that there is support within member societies of EFLM for the concept that Specialists in Laboratory Medicine (SpLM) could provide support for patients in understanding what their test results mean [2]. There have been other calls for such support [3] and experience where patients can access their medical record, which has been positive [4], and the need for appropriate communication of test results to patients has been highlighted using vignettes [5]. Providing test 
results directly to patients has been a key element of the success of electronic communication with patients in the Kaiser Permanente health care system in the USA [6]. In England, there is an active program, conducted through the Department of Health, to enable access by patients to their medical records and laboratory results. In Norway and Denmark, the health care authorities provide the patients access to their medical records through different web portals (e.g. http://helsenorge.no/), but no interpretation of laboratory results is provided. Expressions of concern at patient anxiety are typically expressed by health professionals, although the evidence available does not seem to confirm this is a problem for those willing and able to access their electronic health record [7-10].

The purpose of this approach is to actively include patients in making medical decisions [11, 12]. The goal is that the better informed patient should be empowered to share decision making with health care providers, with improved adherence to treatment, health outcomes and cost-effective health care. Because about two thirds of the clinical decisions are based on laboratory data [13, 14], the access and competent interpretation of laboratory test results seems like an effective approach [15]. Providing adequate information to patients about their laboratory results should enable better concordance and compliance with prescribed medical treatment, better adherence to guidelines and detection and mitigation of errors. Also, it may potentially prevent patients being misled through their searching for information and interpretation for their medical condition from various available, but possibly unreliable, sources.

In order to assess the patients' views on their active involvement in medical decision making by providing access to their laboratory results, with an accompanying commentary and support from the SpLM, EFLM's Patient Focused Laboratory Medicine Working Group have conducted a survey in eight European countries.

\section{Materials and methods}

A questionnaire was devised by one of us (WO) (Supplemental Table 1). The questionnaire underwent institutional ethical review and was distributed to participating members of the Working Group and translated into the local language. Each survey was conducted independently and at different times over a period of 1 year, in part due to local ethical reviews taking time and the logistics of particular sites. Question 5B on fee cost was set at $€ 15$ for The Netherlands and adjusted locally to match the local cost of living. In countries where charges were specifically not allowed, this question was omitted.

A heterogeneous group of 100 consecutive consenting medical clinic outpatients agreed to participate in the survey having received a questionnaire on recruitment and who returned the form at the end of their visit. Results were collated locally and analyzed centrally by two members of the WG (JS, SJ); Denmark and Norway recruited 116 and 367 patients, respectively; the latter was part of a local patient satisfaction survey.

The participating institutions were located in Poland, Serbia, The Netherlands, Denmark, Estonia, Turkey, Norway and Czech Republic. The significantly higher number of participants in Norway may be considered as a limitation of the study and the cause for results' bias. Therefore, we also calculated the mean of the mean (MoM) values for percentages of positive answers in each country. Three countries (Denmark, Czech Republic and Norway) did not ask question 5 on fees as this was incompatible with their health care system; these countries were excluded from data calculations for this question.

We used the $\chi^{2}$-homogeneity test to estimate whether the prevalence of positive answers differed significantly between the eight participating countries. Statistical software SPSS v 20.0 (Chicago, IL, USA) was used for data processing.

\section{Results}

A total of 1084 patients were surveyed, with 100 patients each in Poland, Serbia, Netherlands, Turkey and Czech Republic, 101 in Estonia, 116 in Denmark and 367 in Norway. The summary distribution of answers of all participants is presented in Figure 1. The summary of patients'

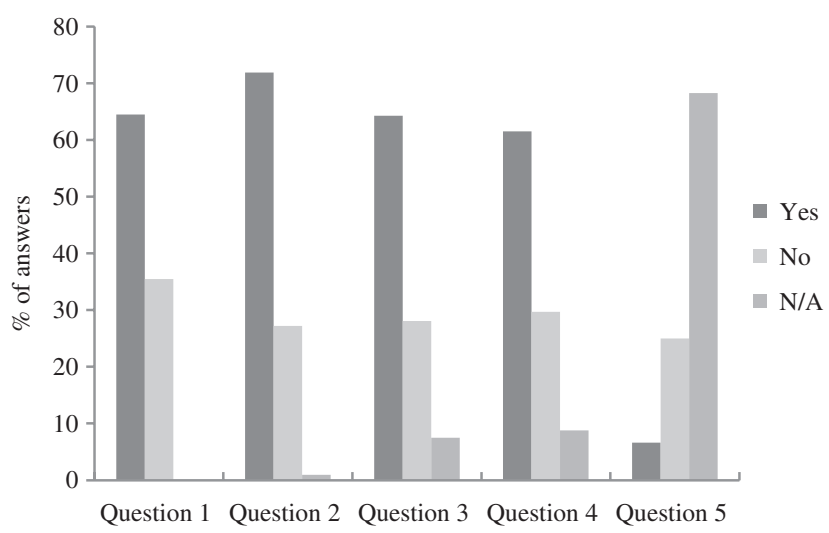

Figure 1: Summary distribution of answers of all participants. Question 1: The laboratory could send the results of the blood tests to you. What is your opinion? Question 2: It often requires special knowledge to understand the results of the laboratory tests. You may therefore need extra information. What is your opinion? Question 3: If you need additional information, would you like to receive this? (Yes = standard description, No = refer to website, N/A = not interested). Question 4: It maybe that you want to receive your report with results, but that the standard of information for you is not enough. A specialist from the laboratory could look at your results and add a personal interpretative comment and explanation. Would you like to receive such a personal report? Question 5: You want a personal commentary and explanation of a laboratory specialist. This extra service takes time and, therefore, cannot be done free of charge. Would you be ready to pay extra for it? 
answers to all five questions in each participating country is presented in Supplemental Table 1.

Of the total of 1084 participating patients, 699 (65\%) wished to receive their own laboratory results and 385 (36\%) did not and were not questioned further. In Serbia, the Netherlands and Estonia, the difference between positive and negative responses was not statistically significant. The other five countries had significantly higher percentage of patients interested in receiving their own laboratory results. There was a significant variation between countries, ranging from $50 \%$ to $94 \%$ wanting their results $(\mathrm{p}<0.0001)$. We identified the countries with the highest (Czech Republic) and lowest (The Netherlands) percentage of positive answers. The contribution of these countries to the overall variability was estimated by comparing the distribution of their answers with the distribution of summary answers of all the remaining countries. With the stepwise analysis, the $\chi^{2}$-test first revealed the significantly higher percentage of positive answers in Czech Republic compared with the summary percentage of the remaining countries $(p<0.0001)$. The following chi-square analysis of the percentage of positive answers between the remaining countries showed that the significant difference remained $(\mathrm{p}=0.0305)$. We then estimated the difference between the distribution of positive answers in the Netherlands and the remaining countries (without Czech Republic, which was excluded in the previous step). The chi-square analysis showed the significantly lower percentage of persons wanting their results in the Netherlands when compared with the remaining countries $(p=0.0128)$. The difference between the other countries was not statistically significant $(p=0.1661)$, indicating that the interest in receiving their own laboratory results was equivalent in the participating countries, when excluding the two countries with extremely high (Czech Republic) and low (The Netherlands) interest.

Of the 699 participants wanting to receive their own laboratory results, 526 (71.9\%) wanted additional information, 199 (27.2\%) felt that they understood the results and $1 \%$ did not respond. The prevalence of positive answers was statistically significantly higher compared to negative answers in all countries but Serbia. The majority of respondents wished for further information, ranging from 59\% to $84 \%$. Chi-square homogeneity test showed no statistically significant difference in distribution of answers in participating countries $(p=0.0507)$. This indicated that those who wanted their own results wanted to receive more information than contained in the standard laboratory report.

When offered options of either having a standard description with information about what the tests meant, or receiving a reference to a website where information about the tests that were done could be found, there was a similar split, with $524(64.4 \%)$ in favor of the former and $229(28.2 \%)$ the latter. Also, $61(7.5 \%)$ participants stated they were not interested in this aspect. Again, there was marked variation between countries $(p<0.0001)$, but a standard description was favored in all cases above the referral to a website. Serbia was the only exception having the largest percentage of respondents who were not interested in receiving any information. However, when Serbia's answers were excluded from the statistical analysis, the difference between countries in the distribution of answers remained statistically significant $(\mathrm{p}<0.0001)$.

When the concept was introduced of having a Specialist in Laboratory Medicine looking at the results, and adding a personal interpretative comment and explanation was introduced, 449 (61.5\%) participants were interested in this, whereas $217(29.7 \%)$ felt that this was not necessary, and 64 (8.8\%) did not respond. Although a personalized report was favored in all countries, a significant minority did not wish such a report. Also, the distribution of answers differed significantly between participating countries $(p<0.0001)$.

The suggestion that comment and interpretation could be added and that there could be a fee to cover the time taken by the SpLM did not meet favor, with only 196 (25.0\%) wanting a personalized report but were not interested in covering the costs and only $52(6.6 \%)$ responding being willing to pay; the majority, 535 (68.3\%), did not respond either way; the difference was pronounced as there were legal and regulatory hurdles to such an approach in Denmark, Norway and Czech Republic reflecting governmental service definitions and fiscal policies. The difference in answer distribution remained statistically significant even when excluding these countries and Serbia from the $\chi^{2}$-analysis $(p<0.0001)$ (Figure 2$)$.

After calculating the MoM values for percentages of positive answers in each country, we obtained almost identical values (i.e. the overall percentage of positive answers for the question 1 was $64.5 \%$, and MoM values in each participating country was $65.1 \%$; for question 2 , the overall mean was $71.9 \%$ vs. MoM of $72.4 \%$; for question 3, $64.4 \%$ vs. $63.3 \%$; and for question $4,61.5 \%$ vs. $63.1 \%$, for overall mean and MoM values, respectively).

\section{Discussion}

The main finding in our study was that there is a clear interest by a significant proportion of patients in receiving individualized comment and interpretation on laboratory 


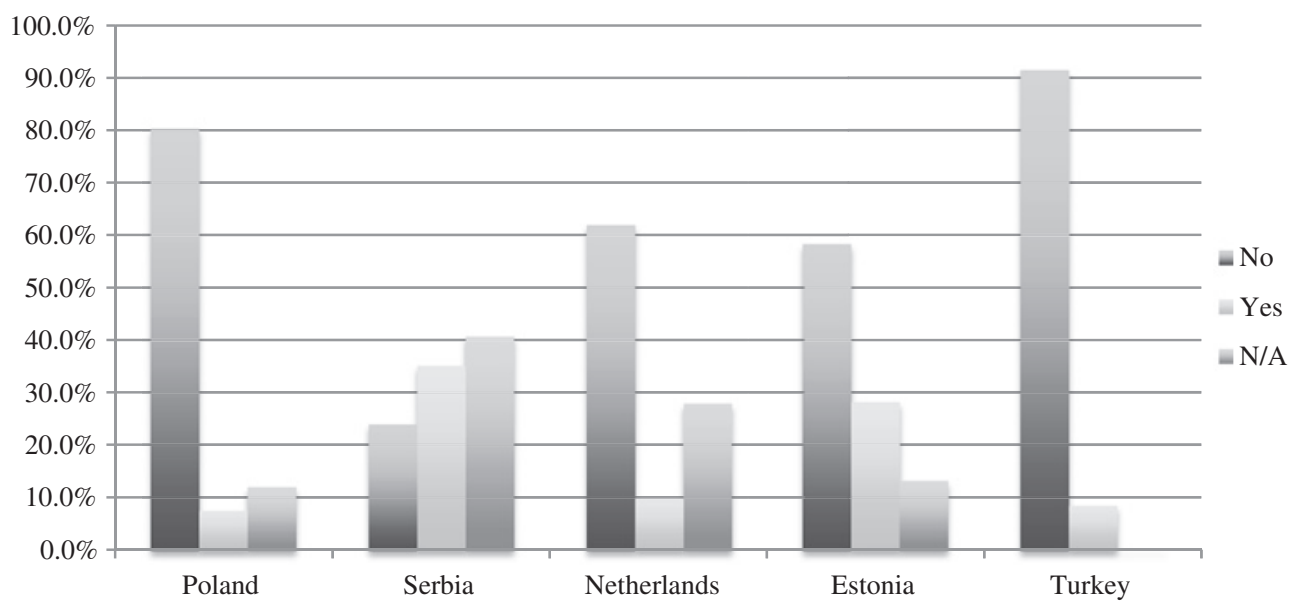

Figure 2: Preferences to pay for an SpLM comment by country (Denmark, Norway and Czech Republic excluded as the option was not permitted).

reports and that SpLM would be acceptable professionals for this purpose. From our work demonstrating a willingness by SpLM professionals to engage in such practice [2], there is an opportunity to progress such an initiative. Patients may want direct information from their clinician [16], but a filtering mechanism, such as through an SpLM, to ensure efficient use of physician time may have value [17], so this must be done in a collaborative approach with the patients' physicians to ensure no confusion or mixed messages ensue, such an approach has been advocated for radiology [18, 19] access to the patient's medical record would enable a better contextual input. From a patient perspective, one can recognize that result immediacy and ownership are factors and such a proposed paradigm may negate the necessity for attendance at the GP surgery, particularly for those with a chronic condition, but this has to be agreed with all parties and these decisions must be respected [20].

The presented results show that, even if the distribution of answers between the countries was not homogenous, there was an overall significant majority of examined patients interested in receiving their results. Also, more than two thirds of patients wanted additional information for interpretation, favoring standardized description within the report over reference to a website. In addition, the majority of participants were interested in receiving a personalized interpretative comment of their results by an SpLM. However, the issue of covering the additional cost of such a service remains open because there are legal and regulatory obstacles in some of the countries for direct charging from the patients. However, even where this is permitted, patients are reluctant to pay, and if they do they place a low monetary value on it, where such information is supplied in larger systems, such an approach is not adopted [21], so there is a perception of added value without causing harm, although evidence to date is anecdotal. Whereas the majority of Serbian participants wanted to receive more information about their results, favoring personalized report, about one third of interested patients were undecided between the three options for receiving additional information (standardized report, website referral or personalized report). This probably caused this group of patients to choose answers "not applicable" for questions 3 and 4. The reason for this situation might be either lack of knowledge, or the opposite - the questioned participants were mainly chronic patients, well informed about their condition, so all three options would be acceptable.

As patients recall of advice and their disease may be poor [22], and may not be up to date, it may be that access to medical files and laboratory results will assist in improving this aspect. Although adding text is the likely means of aiding patient understanding, care has to be taken with any terminology regarding frequency as population numeracy is variable [23], and where possible data presented pictorially or graphically aids comprehension [24], this is particularly true for the elderly and immigrants [24, 25].

Providing such a service, increasing patients' knowledge of their own health, empowers the patient to discover errors in the test-result process. This may be positive as there is evidence that patients play an important role in the detection and mitigation of errors [26].

With the roll-out by health care systems of patientorientated software enabling their access to their medical record, including laboratory results, e.g. My Chart in Denmark, Patient Access in the UK, then the opportunity is there for SpLMs to positively engage in helping patients to better manage their own health care. 
This is an opportunity to make laboratory medicine directly connect with patients, a challenge that needs to be addressed [27]. This will require active engagement by the profession for this to happen, and we would advocate adoption of such an approach. Our working group is developing advice to assist those who wish to proceed [https://www.eflm.eu/upload/docs/Basic\%20 Guidance\%20for\%20PFLM\%202017.pdf (Accessed 7 Mar 2017)].

Acknowledgments: We would like to thank Dr. Patricia Wilkie of the National Association for Patient Participation (UK) and Dr. Amir Hannan, General Practitioner, Haughton Thornley Medical Centre, Manchester, UK, for helpful discussions.

Author contributions: All the authors have accepted responsibility for the entire content of this submitted manuscript and approved submission.

Research funding: None declared.

Employment or leadership: None declared.

Honorarium: None declared.

Competing interests: The funding organization(s) played no role in the study design; in the collection, analysis and interpretation of data; in the writing of the report; or in the decision to submit the report for publication.

\section{References}

1. deLusignan S, Mold F, Sheikh A, Majeed A, Wyatt JC, Quinn T, et al. Patients' online access to their electronic health records and linked online activities: a systematic interpretative review. $\mathrm{Br}$ Med J Open 2014;4:e006021.

2. Watson ID, Siodmiak J, Oosterhuis WP, Corberand J, Jorgensen PE, DikmanZG, et al. European views on patients directly obtaining their laboratory test results. Clin Chem Lab Med 2015;53:1961-6.

3. O'Kane M, Freedman D, Zikmund-Fisher BJ. Can Patients use test results effectively if they have direct access? Br Med J 2015;350:h673.

4. Hannan A. Commentary: UK general practice moves to open notes to patients. Br Med J 2015;350:h266.

5. O'Kane M, Lopez B. Explaining laboratory test results to patients: what the clinician needs to know. Br Med J 2015;351:h5552.

6. Silvestre AL, Sue VM, Allen JY. If you build it will they come? The Kaiser Permanente model of online health care. Health Aff (Millwood) 2009;28:334-44.

7. Delbanco T, Walker J, Darer JD, Elmore JD, Feldman HJ, Leveille SG, et al. Open note: doctors and patients signing on. Ann Intern Med 2005;20:52-7.

8. Delbanco T, Walker J, Bell SK, Darer JD, Elmore JG, Farag N. Inviting patients to read their doctors' notes: a quasi-experimental study and look ahead. Ann Intern Med 2012;157:461-70.

9. Ross SE, Todd J, Moore LA, Beaty BL, Wittevringel L, Lin CT, et al. Expectations of patients and physicians regarding patientaccessible medical records. J Med Internet Res 2006;7:e13.
10. Callen J, Giardina TD, Singh H, Li L, Paoloni R, Georgiou A, et al. Emergency physicians' views of direct notification of laboratory and radiology results to patients using the internet: a multisite survey. J Med Internet Res 2015;17:e60.

11. Moura J, de A Costa BC, de Faria RM, Soares TF, Moura EP, Chiapelli F. Improving communication skill training in patient centered medical practice for enhancing rational use of laboratory tests: the core of bioinformation for leveraging stakeholder engagement in regulatory science. Bioinformation 2013;9:718-20.

12. Cunningham DE, McNab D, Bowie P. Quality and safety issues highlighted by patients in the handling of laboratory test results by general practices-a qualitative study. BMC Health Serv Res 2014;14:206.

13. Plebani M. Exploring the iceberg of errors in laboratory medicine. Clin Chim Acta 2009;404:16-23.

14. Green SF. The cost of poor blood specimen quality and errors in pre-analytical processes. Clin Biochem 2013;46:1175-9.

15. Watson ID. Making test results more easily understood by patients. Br Med J 2015;350:h1942.

16. Lin CT, Wittevrongel L, Moore L, Beaty BL, Ross SE. An internetbased patient-provider communication system: randomized controlled trial. J Med Internet Res 2005;7:e47.

17. http://www.troymedia.com/wp-content/uploads/2012/10/ValuingTimeSaved.pdf. Accessed 23 Feb 2017.

18. Johnson AJ, Frankel RM, Williams LS, Glover S, Easterling D. Patient access to radiology reports: what do physicians think? J Am Coll Radiol 2010;7:281-9.

19. Rubin DL. Informatics methods to enable patient-centered radiology. Acad Radiol 2009;16:524-34.

20. Wilkie P. President of the National Association for Patient Participation (UK) (personal communication). Available at: https:// www.napp.org.uk/index.html. Accessed 27 Feb 2017.

21. Bergmo TS, Wangberg SC. Patients' willingness to pay for electronic communication with their general practitioner. Eur J Health Econ 2007;8:105-10.

22. Hobbs FD, Leif R, Rycroft C. The from the Heart study: a global survey of patient understanding of cholesterol management and cardiovascular risk, and physician-patient communication. Curr Med Res Opin 2008;24:1267-78.

23. Woloshin S, Schwartz LM. Communicating data about the benefits and harms of treatment: a randomized trial. Ann Intern Med 2011;155:87-96.

24. Fuller R, Dudley N, Blacktop J. How Informed is Consent? Understanding of pictorial and verbal probability information by medical inpatients. Postgrad Med 2002;78:543-44.

25. Buetow S, Liew KL, Kenealy T, Dovey S, Elwyn G. Approaches to reducing the most important patient errors in primary health care: patient and professional perspectives. Health Soc Care 2010;18:296-303.

26. Graham DG, Harris DM, Elder NC, Emserman CB, Brandt E, Staton EW, et al. Mitigation of patient harm from testing errors in family medicine offices: a report from the American Academy of Family Physicians National Research Network. Qual Saf Health Care 2008;17:201-8.

27. O'Kane M. Direct patient access to test results: implications for the laboratory. Ann Clin Biochem 2015;52:525-6.

Supplemental Material: The online version of this article (DOI: 10.1515/cclm-2017-0080) offers supplementary material, available to authorized users. 